\title{
BMJ Open Thiazide diuretics and the risk of hip fracture after stroke: a population-based propensity-matched cohort study using Taiwan's National Health Insurance Research Database
}

\author{
Shu-Man Lin, ${ }^{1}$ Shih-Hsien Yang, ${ }^{1,2}$ Hung-Yu Cheng, ${ }^{1}$ Chung-Chao Liang, ${ }^{1}$ \\ Huei-Kai Huang ${ }^{3}$
}

To cite: Lin S-M, Yang S-H, Cheng $\mathrm{H}-\mathrm{Y}$, et al. Thiazide diuretics and the risk of hip fracture after stroke: a population-based propensitymatched cohort study using Taiwan's National Health Insurance Research Database. BMJ Open 2017;7:e016992. doi:10.1136/ bmjopen-2017-016992

- Prepublication history and additional material for this paper are available online. To view please visit the journal (http:// dx.doi.org/10.1136/bmjopen2017-016992).

Received 28 March 2017 Revised 29 June 2017 Accepted 3 July 2017

\section{CrossMark}

${ }^{1}$ Department of Physical Medicine and Rehabilitation, Buddhist Tzu Chi General Hospital, Hualien, Taiwan ${ }^{2}$ School of Medicine, Tzu Chi University, Hualien, Taiwan ${ }^{3}$ Department of Family Medicine, Buddhist Tzu Chi General Hospital, Hualien, Taiwan

Correspondence to Dr Huei-Kai Huang; drhkhuang@gmail.com

\section{ABSTRACT}

Objectives This study aimed to investigate the association between thiazide use and the risk of hip fracture after stroke.

Setting A population-based, propensity-matched cohort study was conducted on the basis of Taiwan's National Health Insurance Research Database.

Participants Patients with newly diagnosed ischaemic stroke between 2000 and 2011 were included. After propensity score matching, 7470 patients were included, of whom 3735 received thiazides and 3735 did not. Outcome measures HRs for developing hip fractures within 2 years after stroke were calculated using Cox proportional hazards regression model with adjustments for sociodemographic and coexisting medical conditions. Results Overall, patients using thiazides after stroke had a lower risk of hip fracture than those not using thiazides (8.5 vs 13.9 per 1000 person-years, adjusted $H R=0.64$, $95 \% \mathrm{Cl} 0.46$ to $0.89, \mathrm{p}=0.007$ ). Further sensitivity analysis based on the duration of thiazide use revealed that the risk of hip fracture tended to decrease as the duration of exposure of thiazides increased. However, the effect was significant only in patients with long-term use of thiazides (using thiazides for $>365$ days within 2 years after stroke), with a $59 \%$ reduction in the risk of hip fracture when compared with patients not using thiazide (adjusted $\mathrm{HR}=0.41,95 \% \mathrm{Cl} 0.22$ to $0.79, \mathrm{p}=0.008$ ).

Conclusions The long-term use of thiazides is associated with a decreased risk of hip fracture after stroke.

\section{INTRODUCTION}

It is well known that hip fracture is associated with an increased risk of major morbidity and mortality. ${ }^{1-3}$ It contributes to disability, morbidity and mortality due to functional loss, and further imposes a considerable socioeconomic burden on society and contributes to deterioration in quality of life. ${ }^{3-5}$ Stroke is a major risk factor for hip fracture that increases the risk of hip fracture by two to four times. ${ }^{6} 7$ Jørgensen $e t a l^{8}$ reported that
Strengths and limitations of this study

- This cohort study was based on a nationwide population database. The findings originated from a representative sample of one million subjects from Taiwan's National Health Insurance Research Database.

- This is the first study to investigate the association between thiazide use and the risk of hip fracture after stroke.

- Some clinical information was not available from the claims database, such as patient lifestyles, as well as physical, psychiatric or laboratory examination data, all of which might be confounding factors.

- Owing to patient anonymity in the National Health Insurance Research Database, we could not directly evaluate the patients to confirm the accuracy of their diagnoses.

the bone mineral density decreased remarkably soon after stroke. In addition, stroke increases the risk of falls; both these conditions predispose to hip fractures ${ }^{9-11}$ and contribute to further decrease in survival rates and reduced recovery of independent mobility after stroke. ${ }^{7}$

More than half the number of patients with stroke had been diagnosed with hypertension before stroke, and hypertension develops after stroke in many patients who were previously normotensive. ${ }^{12-15}$ Thiazide diuretics have been widely used as antihypertensive pharmacological agents for over five decades. ${ }^{16}$ Previous studies have demonstrated that in addition to reducing blood pressure, thiazides modulate calcium homeostasis and are effective in preserving bone mineral density at the hip. ${ }^{17}$ Thiazides reduce urinary calcium excretion, ${ }^{18}$ stimulate osteoblast differentiation and bone mineral 
formation, ${ }^{19}$ and cause metabolic alkalosis ${ }^{20}$ that may lead to decreased bone resorption. ${ }^{21} 22$

Some observational studies have evaluated the relationship between the use of thiazides and the risk of hip fractures. ${ }^{23-28}$ One meta-analysis revealed that thiazides appear to reduce the risk of hip fracture. ${ }^{29}$ However, no current studies on this issue have focused specifically on patients with stroke. It is still unclear whether thiazide use can reduce the risk of hip fracture after stroke, given the profound effects that stroke has on bone mineral density as well as increasing the risk of falls.

Therefore, we conducted a population-based cohort study to investigate whether treatment with thiazides is associated with a reduced risk of hip fracture after stroke.

\section{METHODS}

\section{Data sources}

The source of cohort data set was the National Health Insurance Research Database, Taiwan. In 1995, Taiwan initiated a single-payer insurance programme to finance healthcare, called National Health Insurance (NHI), which covers up to $99 \%$ of Taiwanese population and has contracts with $97 \%$ of hospitals and clinics in Taiwan. ${ }^{30} 31$ The National Health Research Institute of Taiwan has set up a Longitudinal Health Insurance Database for research purposes, by randomly choosing one million NHI beneficiaries. No statistically significant differences were found in the ages, sex or healthcare costs between the one million individuals in the longitudinal database and all NHI beneficiaries. By linking ambulatory and inpatient care claims and the registry of beneficiaries, information about patient demographics and medical records was obtained from the longitudinal database, and used for data analysis. ${ }^{32}$ The data set, which consists of de-identified secondary data for research purposes, was released by the NHI Bureau, and therefore written consent from study subjects was not possible to be obtained.

\section{Study population}

For the present study, we selected the patients with new-onset ischaemic stroke, between years 2000 and 2011, as the study population. These criteria were set as only the patients with ischaemic stroke because claims-based Stroke Severity Index (SSI) (see below) is only applicable to patients with ischaemic stroke, and also because among all the strokes, ischaemic strokes contribute to greater than $80 \% .{ }^{33}$ The study patients were selected on the basis of primary discharge diagnosis of ischaemic stroke (International Classification of Diseases, 9th Revision, Clinical Modification (ICD-9-CM) codes 433 and 434). We defined the concurrent hospitalisation and the date of new-onset stroke, respectively, as the index hospitalisation and the index date, in accordance with our earlier study. ${ }^{32}$ The exclusion criteria were as follows: (1) age <20years; (2) history of stroke before the year 2000; (3) history of hip fracture before the index date; (4) simultaneous hip fracture diagnosis during index hospitalisation; and (5) mortality during index hospitalisation.

\section{Exposure to thiazides}

To identify patients taking thiazides, we identified all prescriptions for thiazides among the study population within 2 years after the date of stroke diagnosis. Patients who were prescribed thiazides during follow-up period were defined as the thiazide cohort; patients who were not prescribed thiazides were defined as the non-thiazide cohort. Sensitivity analysis was further performed to evaluate the effect of duration of exposure. The thiazide cohort was divided into three subgroups according to the duration of thiazide use within 2 years after stroke: $1-90$ days, $91-365$ days and $>365$ days.

\section{Primary outcome}

Identification of patients diagnosed with new hip fractures, within 2 years after stroke, was accomplished using ICD-9-CM codes of 820.x if patients were hospitalised for hip fracture. The validity of diagnosis of hip fracture according to a hospital inpatient assessment is considered acceptable. ${ }^{34}$ All subjects were followed from the index date until a new diagnosis of hip fracture, death or 2 years after stroke. Death was defined as the date a patient was withdrawn from the Taiwan NHI programme. ${ }^{35} 36$ The date of withdrawal from the NHI programme has been recognised as an accurate and reliable proxy for date of mortality. ${ }^{37} 38$

\section{Covariates and propensity score matching}

Baseline characteristics and clinical details of all the study patients were obtained using the ICD-9-CM and procedure codes from both outpatient and inpatient reimbursement claims. Information from each patient's pre-existing clinical condition was used to calculate Charlson Comorbidity Index scores. ${ }^{39}$ For proxy indicators to represent stroke severity, data related to the index hospitalisation, including diagnosis codes for hemiplegia, paraplegia and aphasia or operation, as well as procedure codes for mechanical ventilation and intensive care unit utilisation, were collected. ${ }^{40}$ The SSI score was also calculated to predict the severity of the neurological deficit. The claims-based SSI developed by Sung $e t a t^{11}$ is highly correlated with the National Institutes of Health Stroke Scale. The baseline comorbidities and use of medications which were considered as possible potential confounders (listed in table 1) were selected according to previous studies. ${ }^{42-48}$ The criteria for defining pre-existing comorbidity was the positive diagnosis of any disease either at the time of a minimum of one hospital admission or two outpatient visits within the year prior to the index date. A baseline use of medication was defined as the use of these drugs at least 30 days during the year preceding the index date. The use of osteoporosis medication was defined as any prescription of medication including bisphosphonates, hormone replacement therapy, calcitonin or vitamin D supplementation available in Taiwan. ${ }^{49}$ 
Table 1 Baseline characteristics of patients with stroke according to thiazide use after propensity score matching

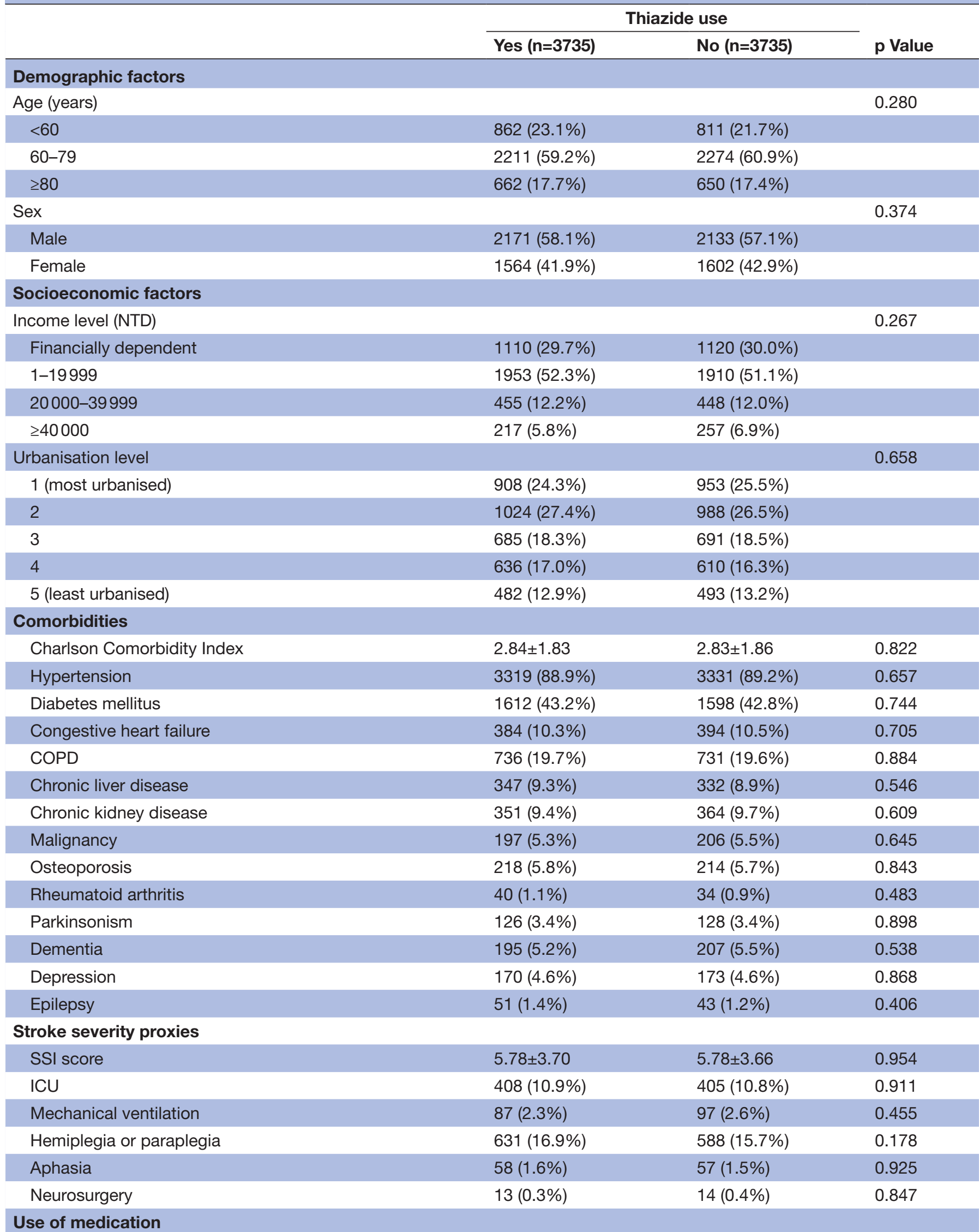

Antihypertensive drugs other than thiazides 
Table 1 Continued

\begin{tabular}{|c|c|c|c|}
\hline & \multicolumn{2}{|c|}{ Thiazide use } & \multirow[b]{2}{*}{ p Value } \\
\hline & Yes $(n=3735)$ & No $(n=3735)$ & \\
\hline $\mathrm{ACEl}, \mathrm{ARB}$ & $1543(41.3 \%)$ & $1539(41.2 \%)$ & 0.925 \\
\hline$\beta$-blockers & $1128(30.2 \%)$ & $1158(31.0)$ & 0.451 \\
\hline Loop diuretics & $318(8.5 \%)$ & $325(8.7 \%)$ & 0.773 \\
\hline Anxiolytics, antipsychotics, sedatives or antidepressants & $1164(31.2 \%)$ & $1184(31.7 \%)$ & 0.618 \\
\hline Systemic corticosteroids & $225(6.0 \%)$ & $238(6.4 \%)$ & 0.533 \\
\hline Osteoporosis medication & $130(3.5 \%)$ & $130(3.5 \%)$ & 1.000 \\
\hline
\end{tabular}

Continuous data expressed as mean \pm SD and categorical data expressed as number (\%).

ACEI, ACE inhibitors; ARB, angiotensin receptor blocker; COPD, chronic obstructive pulmonary disease; ICU, intensive care unit; NTD, New Taiwan dollars; SSI, Stroke Severity Index.

Registration and claim records of 1,000,000 individuals in the National Health Insurance Research Database
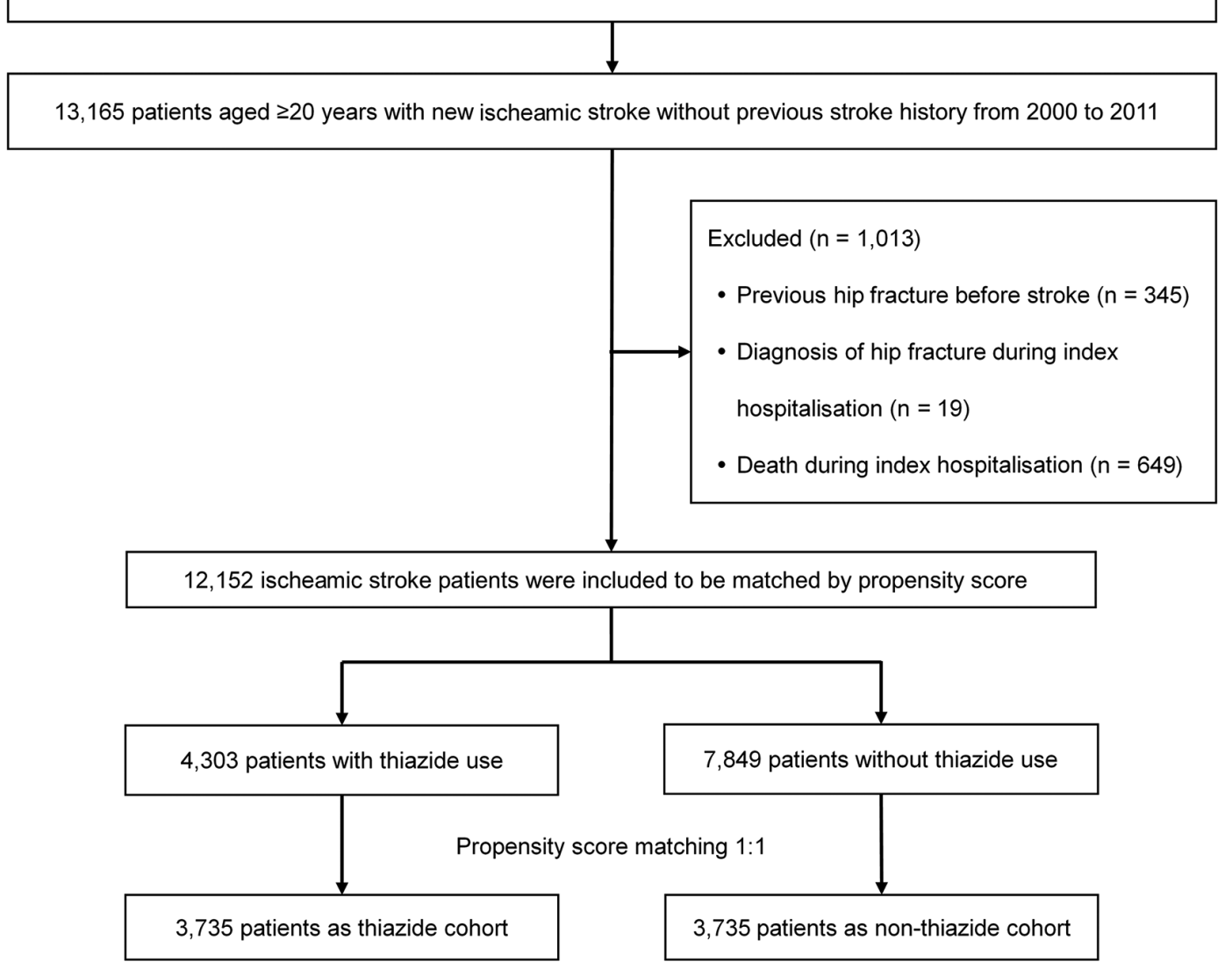

Figure 1 Flow diagram of the selection of study subjects.

NHI insurance premiums are based on income level. To assess socioeconomic status, the premiums paid were used as a proxy for the income level of patients and classified into four strata (New Taiwan dollars $\geq 40000$, 20000-39 999, 1-19 999 and financially dependent). The unemployed, students, children and elderly individuals without a salary are designated as 'fixed amount' (ie, dependents) by the Bureau of National Health Insurance. Their insurance premiums are covered by the government or their foster families. ${ }^{50}$ The location of included patients' living quarters, obtained from NHI database, was also used to stratify them in different levels. Urbanisation levels in Taiwan are divided into seven levels. Level 1 represents the most urbanised area and level 7 represents the least urbanised. ${ }^{51}{ }^{52}$ To simplify the analysis, levels 5 through 7 were combined into a single group and are hereafter referred to as level 5 . 


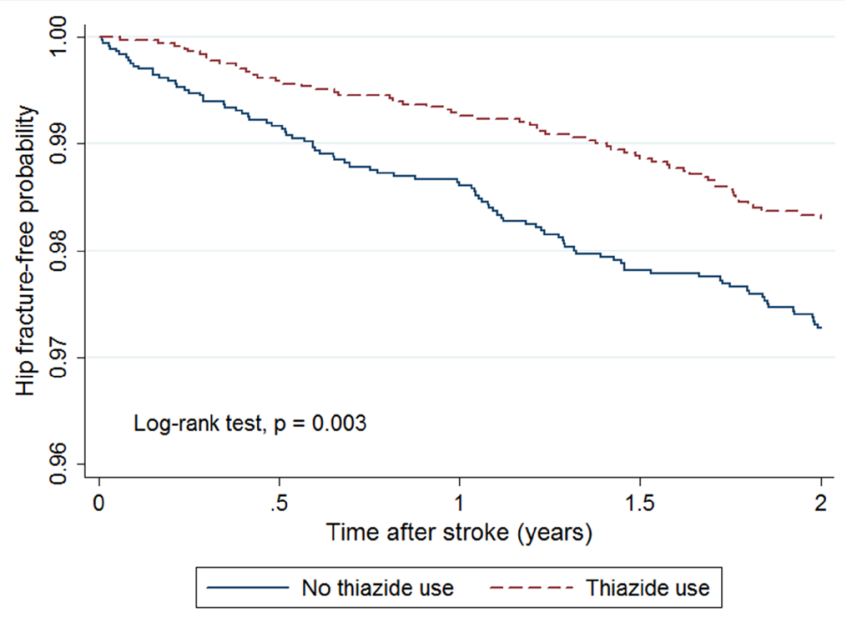

Figure 2 Kaplan-Meier curves showing estimated hip fracture-free probability of patients after stroke according to the use of thiazides.

Table 2 Risk of hip fracture for patients with stroke according to thiazide use

\begin{tabular}{|c|c|c|}
\hline & \multicolumn{2}{|l|}{ Thiazide use } \\
\hline & Yes & No \\
\hline Patients (n) & 3735 & 3735 \\
\hline Hip fractures & 60 & 92 \\
\hline Person-years & 7074.9 & 6596.4 \\
\hline Incidence rate* & 8.5 & 13.9 \\
\hline \multicolumn{3}{|l|}{ Univariate model } \\
\hline Crude HR (95\% Cl) & 0.65 (0.47 to 0.90$)$ & 1 (ref) \\
\hline p Value & 0.009 & \\
\hline \multicolumn{3}{|l|}{ Multivariate model† } \\
\hline Adjusted HR (95\% Cl) & 0.64 (0.46 to 0.89$)$ & 1 (ref) \\
\hline $\mathrm{p}$ Value & 0.007 & \\
\hline
\end{tabular}

*Per 1000 person-years.

†Multivariate Cox proportional hazard regression model, adjusting for all baseline characteristics (listed in table 1) and competing mortality.

Necessary care was taken to decrease the patient selection bias, by matching the propensity score in order to balance baseline differences and covariates, and these include age, sex, stroke severity proxies, socioeconomic factors, baseline comorbidities and medication use (see table 1). For each thiazide user and non-user, a propensity score was calculated to estimate the probability of thiazide use by logistic regression model with all baseline covariates. Propensity score similarity using a calliper width equal to 0.2 of SD of the logit of the score, on the basis of nearest-neighbour matching, was used to match each thiazide user with a non-thiazide user. $^{53} 54$ In addition, to eliminate the possibility of different study results between performing and not performing propensity score matching, we also conducted sensitivity analyses that included all patients without matching.

\section{Statistical analysis}

Results are calculated and presented as mean $\pm \mathrm{SD}$ for continuous variables and as percentages for categorical variables. To evaluate differences between the thiazide and non-thiazide cohorts, a t test was used to analyse continuous variables and a $\chi^{2}$ contingency table analysis for categorical variables. We used the Kaplan-Meier method to estimate the hip fracture-free rates and the log-rank test to compare differences between the survival curves. The HRs with $95 \%$ CIs for the association of hip fracture with thiazide use were calculated using univariate and multivariate Cox proportional hazards regression models, after adjustment for the baseline characteristics listed in table 1. Cox proportional hazards regression models were performed in the presence of competing risk event with adjustment for competing mortality. ${ }^{355}$ Statistical analyses were conducted using Stata V.13. Results were considered significantly different when the two-sided probability value was $<0.05$.

\section{RESULTS \\ Demographic characteristics of subjects}

After propensity score matching, 7470 patients with ischaemic stroke without previous hip fracture were included in our study. Among these patients, 3735 received thiazides and 3735 did not (figure 1). All baseline characteristics were well balanced between the two cohorts. There were no significant differences in age, sex, income levels, urbanisation levels, baseline comorbidities, stroke severity proxies or use of medications (table 1). During the follow-up period, 987 patients died, which includes 369 deaths in the thiazide cohort and 618 deaths in the non-thiazide cohort.

Table 3 Risk of hip fracture for patients with stroke according to the duration of thiazide use

\begin{tabular}{|c|c|c|c|c|c|c|c|c|}
\hline \multirow{2}{*}{$\begin{array}{l}\text { Duration of } \\
\text { thiazide use }\end{array}$} & \multirow[b]{2}{*}{ Patients (n) } & \multirow[b]{2}{*}{ Hip fractures } & \multirow{2}{*}{$\begin{array}{l}\text { Person- } \\
\text { years }\end{array}$} & \multirow{2}{*}{$\begin{array}{l}\text { Incidence } \\
\text { rate }^{\star}\end{array}$} & \multicolumn{2}{|l|}{ Univariate model } & \multicolumn{2}{|l|}{ Multivariate model† } \\
\hline & & & & & Crude HR $(95 \% \mathrm{Cl})$ & p Value & Adjusted HR (95\% CI) & p Value \\
\hline Non-user & 3735 & 92 & 6596.4 & 13.9 & 1 (ref) & & 1 (ref) & \\
\hline $1-90$ days & 1488 & 28 & 2710.1 & 10.3 & 0.76 (0.50 to 1.16$)$ & 0.206 & 0.73 (0.48 to 1.13$)$ & 0.160 \\
\hline 91-365 days & 1296 & 22 & 2477.5 & 8.9 & 0.69 (0.43 to 1.09$)$ & 0.111 & $0.69(0.43$ to 1.11$)$ & 0.127 \\
\hline$>365$ days & 951 & 10 & 1887.3 & 5.3 & $0.42(0.22$ to 0.81$)$ & 0.010 & 0.41 (0.22 to 0.79$)$ & 0.008 \\
\hline
\end{tabular}

*Per 1000 person-years.

†Multivariate Cox proportional hazard regression model, adjusting for all baseline characteristics (listed in table 1) and competing mortality. 


\section{Overall analysis depending on thiazide use}

Kaplan-Meier survival analysis showed a lower cumulative incidence of hip fracture during the 2-year follow-up in the thiazide cohort than in the non-thiazide cohort $(8.5$ vs 13.9 per 1000 person-years; log-rank test, $\mathrm{p}=0.003$; figure 2). The average time from index date to hip fracture for patients who developed hip fracture was 1.09 years in thiazide cohort and 0.89 years in non-thiazide cohort. After calculating the HRs by Cox regression models, we found that thiazide use after stroke was associated with a significantly lower risk in both univariate (crude $\mathrm{HR}=0.65,95 \%$ CI 0.47 to $0.90, \mathrm{p}=0.009$ ) and multivariate Cox regression models (adjusted $\mathrm{HR}=0.64$, $95 \%$ CI to $0.46-0.89, \mathrm{p}=0.007$ ) (table 2 ). Sensitivity analysis conducted without propensity score matching also revealed similar results (see online supplementary table S1).

\section{Sensitivity analysis based on the duration of thiazide use}

Sensitivity analysis was performed to examine whether the duration of thiazide use affected hip fracture risk (table 3). The average time from index date to hip fracture was 0.99 years in patients using thiazides for 1-90 days, 1.08 years in those using thiazides for 91-365 days, and 1.39 years in those using thiazides for $>365$ days. Compared with non-users, long-term thiazide users (taking the drug $>365$ days within 2 years after stroke) had a significantly lower risk of hip fracture (crude $\mathrm{HR}=0.42$, $95 \%$ CI 0.22 to $0.81, \mathrm{p}=0.010$; adjusted $\mathrm{HR}=0.41,95 \%$ CI 0.22 to $0.79, \mathrm{p}=0.008)$. Although no significant effect was observed in the two subgroups with shorter exposure durations, the hip fracture risk tended to be lower as the duration of exposure increased according to the value of estimated HRs (table 3). Sensitivity analysis conducted without propensity score matching also revealed similar results (see online supplementary table S2). The baseline characteristics of subgroups according to the duration of thiazide use are shown in online supplementary table S3.

\section{DISCUSSION}

In this population-based, propensity-matched cohort study, we found that long-term thiazide use was associated with a reduced risk of hip fracture after stroke. To the best of the authors' knowledge, this is the first study to evaluate the relationship of thiazide use and the risk of hip fracture after stroke.

Both stroke and hip fracture are associated with significant disability, morbidity and mortality. ${ }^{69}{ }^{56}$ People who suffered stroke are at much higher risk of developing hip fractures within 2 years following stroke, as compared with general population without stroke. ${ }^{11}{ }^{57}$ Therefore, the prevention of hip fracture is an important clinical issue for patients after stroke. Several studies have summarised the need to focus on preventing falls and avoiding loss of bone mineral density in order to prevent hip fracture after stroke. 7115859
Although there are concerns that diuretics may increase the risk of fall in the elderly, hence increasing the fracture risk, there is evidence to support the notion that thiazides may actually prevent hip fracture by reducing the loss of bone mineral density, as these drugs decrease excretion of calcium through urine. ${ }^{17242628}$ A meta-analysis based on cohort studies showed that thiazide use was associated with a reduction in risk of hip fracture by $24 \%$ (pooled risk ratio $=0.76) .{ }^{29}$ One large-scale, case-control study revealed that only the long-term use of thiazides was associated with a reduction of $19 \%$ in the risk of hip fracture $(\mathrm{OR}=0.81) .{ }^{28} \mathrm{In}$ addition, a recent secondary analysis of a randomised clinical trial revealed a reduction of $21 \%$ in the risk of hip fracture with the use of thiazides in patients with hypertension $(\mathrm{HR}=0.79) .{ }^{60}$ However, none of these studies specifically assessed patients after stroke. The effect of reducing hip fracture by thiazide use in patients after stroke had not previously been evaluated. Our study focused on this particular population to fill this gap in our knowledge, and demonstrated that long-term use of thiazides (using thiazides for $>365$ days in the 2 years following stroke) can significantly reduce hip fracture risk after stroke, with a reduction in fracture risk of $59 \%$ (adjusted HR=0.41). The effect of reducing hip fracture by thiazide use seems to be more pronounced in our study of patients after stroke than in previous studies focused on the general population. This may be explained by the protective effect of thiazides against the rapid decrease in bone mineral density after stroke.

The following are the strengths of our study: (1) The main strength comes from the fact that this study is highly representative of Taiwan's population as the study employs a representative sample of one million people from a nationwide database, National Health Insurance Research Database. (2) Being a cohort study, the level of evidence generated in the present study is of higher level, as compared with case-control or cross-sectional studies. (3) In this study, we used propensity score matching to balance between thiazide users and non-users, in order to avoid any influence of baseline demographic and clinical characteristics of the included patients. Further, multivariate Cox proportional hazard regression models assisted in controlling for residual confounding factors that might affect the relationship between hip fractures and thiazide use.

Nevertheless, our study has several limitations. First, some clinical information was not available from the claims-based data, such as patient lifestyles, as well as physical, psychiatric or laboratory examination data, all of which might be confounding factors for hip fracture. In addition, bias related to adjustment for confounders might still be present in a cohort study. It is important to keep in mind that bias because of unknown and unmeasured confounding factors may exist even after including sufficient controls for the known confounding factors. Second, determination of the severity of stroke and its grading using stroke severity proxies is not the most appropriate way. Nevertheless, the claims-based SSI calculated 
as a representative of neurological deficit matched well between two cohorts after tallying their propensity scores. A previous study on outcomes research using administrative data indicated that the SSI is a potential proxy indicator for ischaemic stroke severity. ${ }^{41}$ There is a significant correlation between the SSI and clinical stroke severity, scored in accordance to the National Institutes of Health Stroke Scale, and also there is significant association between SSI and the modified Rankin Scale, which is used for follow-up evaluations. ${ }^{61}$ Therefore, differences in stroke severity between the thiazide and non-thiazide cohorts were diminished after adequate matching. Finally, the NHI claim records are not for scientific analyses initially and are meant primarily for administrative use, and also because the patient data are anonymous, there is no way to directly confirm patients' diagnoses. ${ }^{62}$ However, subjects were identified only when they were hospitalised for stroke or hip fracture, so that the validity of diagnosis of stroke and hip fracture according to claims data from the hospital inpatient assessment should be acceptable.

\section{CONCLUSION}

In summary, this population-based, propensity scorematched, cohort study indicated that long-term use of thiazides is associated with a decreased hip fracture risk after stroke. Further prospective randomised trials are needed to confirm this finding.

Acknowledgements The authors are grateful to Jen-Hung Wang for advice in statistics.

Contributors Study conception and design: S-ML, S-HY and H-KH; acquisition of data: S-HY and H-KH; analysis and interpretation of data: S-ML, S-HY, H-YC, C-CL and $\mathrm{H}-\mathrm{KH}$; drafting of manuscript: S-ML and $\mathrm{H}-\mathrm{KH}$; critical revision: S-ML, S-HY, $\mathrm{H}-\mathrm{YC}, \mathrm{C}-\mathrm{CL}$ and $\mathrm{H}-\mathrm{KH}$.

\section{Competing interests None declared.}

Ethics approval The study protocol was approved by the Institutional Review Board of Tzu Chi Medical Center (REC No: IRB104-131C).

Provenance and peer review Not commissioned; externally peer reviewed.

Data sharing statement All relevant data are within the paper. No additional data are available.

Open Access This is an Open Access article distributed in accordance with the Creative Commons Attribution Non Commercial (CC BY-NC 4.0) license, which permits others to distribute, remix, adapt, build upon this work non-commercially, and license their derivative works on different terms, provided the original work is properly cited and the use is non-commercial. See: http://creativecommons.org/ licenses/by-nc/4.0/

(c) Article author(s) (or their employer(s) unless otherwise stated in the text of the article) 2017. All rights reserved. No commercial use is permitted unless otherwise expressly granted.

\section{REFERENCES}

1. Cummings SR, Melton LJ. Epidemiology and outcomes of osteoporotic fractures. Lancet 2002;359:1761-7.

2. Bentler SE, Liu L, Obrizan M, et al. The aftermath of hip fracture: discharge placement, functional status change, and mortality. Am J Epidemiol 2009;170:1290-9.

3. Farahmand BY, Michaëlsson K, Ahlbom A, et al. Survival after hip fracture. Osteoporos Int 2005;16:1583-90.
4. Randell AG, Nguyen TV, Bhalerao N, et al. Deterioration in quality of life following hip fracture: a prospective study. Osteoporos Int 2000;11:460-6.

5. Braithwaite RS, Col NF, Wong JB. Estimating hip fracture morbidity, mortality and costs. J Am Geriatr Soc 2003;51:364-70.

6. Luan L, Li R, Wang Z, et al. Stroke increases the risk of hip fracture: a systematic review and meta-analysis. Osteoporos Int 2016;27:3149-54.

7. Ramnemark A, Nyberg L, Borssén B, et al. Fractures after stroke. Osteoporos Int 1998;8:92-5.

8. Jørgensen L, Engstad T, Jacobsen BK. Bone mineral density in acute stroke patients: low bone mineral density may predict first stroke in women. Stroke 2001;32:47-51.

9. Carda S, Cisari C, Invernizzi M, et al. Osteoporosis after stroke: a review of the causes and potential treatments. Cerebrovasc Dis 2009;28:191-200.

10. Ramnemark A, Nilsson M, Borssén B, et al. Stroke, a major and increasing risk factor for femoral neck fracture. Stroke 2000;31:1572-7.

11. Dennis MS, Lo KM, McDowall M, et al. Fractures after stroke: frequency, types, and associations. Stroke 2002;33:728-34.

12. Harrington C. Managing hypertension in patients with stroke. Are you prepared for labetalol infusion? Crit Care Nurse 2003;23:30-8.

13. Shih CC, Liao CC, Sun MF, et al. A Retrospective Cohort Study Comparing Stroke Recurrence Rate in Ischemic Stroke Patients With and Without Acupuncture Treatment. Medicine 2015;94:e1572.

14. Takahashi T, Harada M, Kikuno T, et al. Prevalence of metabolic syndrome in stroke patients: a prospective multicenter study in Japan. Acute Med Surg 2014;1:17-22.

15. Hsieh Fl, Chiou HY. Stroke: morbidity, risk factors, and care in taiwan. J Stroke 2014;16:59-64.

16. Musini VM, Nazer M, Bassett K, et al. Blood pressure-lowering efficacy of monotherapy with thiazide diuretics for primary hypertension. Cochrane Database Syst Rev 2014;5:CD003824.

17. LaCroix AZ, Ott SM, Ichikawa L, et al. Low-dose hydrochlorothiazide and preservation of bone mineral density in older adults. A randomized, double-blind, placebo-controlled trial. Ann Intern Med 2000;133:516-26.

18. Middler S, Pak CY, Murad F, et al. Thiazide diuretics and calcium metabolism. Metabolism 1973;22:139-46.

19. Dvorak MM, De Joussineau C, Carter DH, et al. Thiazide diuretics directly induce osteoblast differentiation and mineralized nodule formation by interacting with a sodium chloride co-transporter in bone. J Am Soc Nephrol 2007;18:2509-16.

20. Miltiadous G, Mikhailidis DP, Elisaf M. Acid-base and electrolyte abnormalities observed in patients receiving cardiovascular drugs. $J$ Cardiovasc Pharmacol Ther 2003;8:267-76.

21. Arnett TR, Spowage M. Modulation of the resorptive activity of rat osteoclasts by small changes in extracellular $\mathrm{pH}$ near the physiological range. Bone 1996;18:277-9.

22. Pierce WM, Nardin GF, Fuqua MF, et al. Effect of chronic carbonic anhydrase inhibitor therapy on bone mineral density in white women. J Bone Miner Res 1991;6:347-54.

23. Cauley JA, Cummings SR, Seeley DG, et al. Effects of thiazide diuretic therapy on bone mass, fractures, and falls. The Study of Osteoporotic Fractures Research Group. Ann Intern Med 1993;118:666-73.

24. Feskanich D, Willett WC, Stampfer MJ, et al. A prospective study of thiazide use and fractures in women. Osteoporos Int 1997;7:79-84.

25. Guo Z, Wills $P$, Viitanen $M$, et al. Cognitive impairment, drug use, and the risk of hip fracture in persons over 75 years old: a communitybased prospective study. Am J Epidemiol 1998;148:887-92.

26. LaCroix AZ, Wienpahl J, White LR, et al. Thiazide diuretic agents and the incidence of hip fracture. N Engl J Med 1990;322:286-90.

27. Schoofs MW, van der Klift M, Hofman A, et al. Thiazide diuretics and the risk for hip fracture. Ann Intern Med 2003;139:476-82.

28. Rejnmark L, Vestergaard P, Mosekilde L. Reduced fracture risk in users of thiazide diuretics. Calcif Tissue Int 2005;76:167-75.

29. Aung K, Htay T. Thiazide diuretics and the risk of hip fracture. Cochrane Database Syst Rev 2011;10:CD005185.

30. Chen HF, Lee SP, Li CY. Sex differences in the incidence of hemorrhagic and ischemic stroke among diabetics in Taiwan. $J$ Womens Health 2009;18:647-54.

31. Chou YC, Liao CC, Su LT, et al. Stroke rehabilitation is associated with a reduction in dementia risk: a population-based retrospective cohort study. J Rehabil Med 2012;44:319-24.

32. Huang HK, Lin SM, Yang CSH, et al. Post-ischemic stroke rehabilitation is associated with a higher risk of fractures in older women: A population-based cohort study. PLoS One 2017;12:e0175825. 
33. Andersen KK, Olsen TS, Dehlendorff C, et al. Hemorrhagic and ischemic strokes compared: stroke severity, mortality, and risk factors. Stroke 2009;40:2068-72.

34. Wu CH, Liou TH, Hsiao PL, et al. Contribution of ischemic stroke to hip fracture risk and the influence of gender difference. Arch Phys Med Rehabil 2011;92:1987-91.

35. Wu CY, Chen YJ, Ho HJ, et al. Association between nucleoside analogues and risk of hepatitis $B$ virus-related hepatocellular carcinoma recurrence following liver resection. JAMA 2012;308:1906-14.

36. Su TH, Chang SH, Chen PC, et al. Temporal Trends in Treatment and Outcomes of Acute Myocardial Infarction in Patients With Chronic Obstructive Pulmonary Disease: A Nationwide Population-Based Observational Study. J Am Heart Assoc 2017;6.

37. Lien HM, Chou SY, Liu JT. Hospital ownership and performance: evidence from stroke and cardiac treatment in Taiwan. $J$ Health Econ 2008;27:1208-23.

38. Cheng CL, Chien HC, Lee $\mathrm{CH}$, et al. Validity of in-hospital mortality data among patients with acute myocardial infarction or stroke in National Health Insurance Research Database in Taiwan. Int J Cardiol 2015;201:96-101.

39. Charlson ME, Pompei P, Ales KL, et al. A new method of classifying prognostic comorbidity in longitudinal studies: development and validation. J Chronic Dis 1987;40:373-83.

40. Lee HC, Chang KC, Huang YC, et al. Inpatient rehabilitation utilization for acute stroke under a universal health insurance system. Am J Manag Care 2010;16:e67-e74.

41. Sung SF, Hsieh CY, Kao Yang YH, et al. Developing a stroke severity index based on administrative data was feasible using data mining techniques. J Clin Epidemiol 2015;68:1292-300.

42. Campbell AJ, Robertson MC. Implementation of multifactorial interventions for fall and fracture prevention. Age Ageing 2006;35(Suppl 2)ii60-4.

43. Kessel B. Hip fracture prevention in postmenopausal women. Obstet Gynecol Surv 2004;59:446-55. quiz 85.

44. Huang SW, Wang WT, Chou LC, et al. Chronic Obstructive Pulmonary Disease Increases the Risk of Hip Fracture: A Nationwide Population-Based Cohort Study. Sci Rep 2016;6:23360.

45. Huusko TM, Korpela M, Karppi P, et al. Threefold increased risk of hip fractures with rheumatoid arthritis in Central Finland. Ann Rheum Dis 2001;60:521-2.

46. Culafić D, Djonic D, Culafic-Vojinovic V, et al. Evidence of degraded BMD and geometry at the proximal femora in male patients with alcoholic liver cirrhosis. Osteoporos Int 2015;26:253-9.

47. Kim SM, Long J, Montez-Rath M, et al. Hip Fracture in Patients With Non-Dialysis-Requiring Chronic Kidney Disease. J Bone Miner Res 2016;31:1803-9.
48. Cummings SR, Eastell R. Risk and Prevention of Fracture in Patients With Major Medical Illnesses: A Mini-Review. J Bone Miner Res 2016;31:2069-72.

49. Hwang JS, Chan DC, Chen JF, et al. Clinical practice guidelines for the prevention and treatment of osteoporosis in Taiwan: summary. $J$ Bone Miner Metab 2014;32:10-16.

50. Wu JC, Liu L, Wen-Cheng $\mathrm{H}$, et al. The incidence of adjacent segment disease requiring surgery after anterior cervical diskectomy and fusion: estimation using an 11-year comprehensive nationwide database in Taiwan. Neurosurgery 2012;70:594-601.

51. Lin HC, Chao PZ, Lee HC. Sudden sensorineural hearing loss increases the risk of stroke: a 5-year follow-up study. Stroke 2008;39:2744-8.

52. Liu CY HY, Chuang YL, Chen YJ, et al. Liu JS Liang KY. Incorporating development stratification of Taiwan townships into sampling design of large scale health interview survey (in Chinese). J Health Manage 2006;4:1-22.

53. Leuven E, Sianesi B. PSMATCH2: Stata module to perform full Mahalanobis and propensity score matching, common support graphing, and covariate imbalance testing. 2003 http://ideas.repec. org/c/boc/bocode/s432001.html (accessed 5 May 2017).

54. Chang CC, Chen YT, Hsu CY, et al. Dipeptidyl Peptidase-4 Inhibitors, Peripheral Arterial Disease, and Lower Extremity Amputation Risk in Diabetic Patients. Am J Med 2017;130:348-55.

55. Fine JP, Gray RJ. A Proportional Hazards Model for the Subdistribution of a Competing Risk. J Am Stat Assoc 1999;94:496-509.

56. Andersson AG, Seiger A, Appelros P. Hip fractures in persons with stroke. Stroke Res Treat 2013;2013:1-7.

57. Kanis J, Oden A, Johnell O. Acute and long-term increase in fracture risk after hospitalization for stroke. Stroke 2001;32:702-6.

58. Myint PK, Poole KE, Warburton EA. Hip fractures after stroke and their prevention. QJM 2007;100:539-45.

59. Pouwels S, Lalmohamed A, Leufkens B, et al. Risk of hip/femur fracture after stroke: a population-based case-control study. Stroke 2009;40:3281-5.

60. Puttnam R, Davis BR, Pressel SL, et al. Association of 3 Different Antihypertensive Medications With Hip and Pelvic Fracture Risk in Older Adults: Secondary Analysis of a Randomized Clinical Trial. JAMA Intern Med 2017;177:67-76.

61. Sung SF, Hsieh CY, Lin HJ, et al. Validity of a stroke severity index for administrative claims data research: a retrospective cohort study. BMC Health Serv Res 2016;16:509.

62. Harnod T, Wang YC, Kao CH. Association of Migraine and SleepRelated Breathing Disorder: A Population-Based Cohort Study. Medicine 2015;94:e1506. 